\title{
La réhabilitation buccale implantaire chez l'enfant
}

\section{Oral implant by young patient}

SIHAM TAISSE, SAMIRA EL ARABI, ASMA KHRIBCHI, KARIM IDRISSI-KAITOUNI, IHSSANE BENYAHYA

\begin{abstract}
RÉSUMÉ
L'absence de dents consécutive à un traumatisme, I'oligodontie ou l'anodontie sévère chez l'enfant nécessitent une réhabilitation buccale précoce. En effet ces anomalies dentaires peuvent avoir des conséquences sur le développement craniofacial et mental de l'enfant et s'accompagner de troubles fonctionnels et esthétiques qui s'aggravent à l'âge adulte ; d'où la nécessité d'une thérapeutique prothétique pour restaurer les différentes fonctions perturbées.

L'objectif de cet article est de discuter l'éventualité de la réhabilitation buccale implantaire chez l'enfant, ainsi que l'influence de la croissance osseuse et dentaire des maxillaires sur la stabilité des implants dentaires.

Il est généralement recommandé de différer la réhabilitation buccale implantaire jusqu'à l'achèvement de la croissance osseuse et dentaire, sauf dans les cas d'anodontie ou d'oligodontie sévère associées à des syndromes complexes. Med Buccale Chir Buccale 2007; 13 : 219-222.
\end{abstract}

mots clés: enfant, implant, croissance, réhabilitation orale, dysplasie ectodermique.

\section{SUMMARY}

The absence of teeth consecutive to a trauma, oligodonty or severe anodontia in the child requires an early buccal rehabilitation. As a matter of fact, these dental anomalies may have repercussions on the craniofacial and mental development of children and may be accompanied by functional and esthetic disorders which get worse at adult age, whence the necessity of undertaking a prosthetic therapy to restore the various dysfunctions.

The objective of this article is to discuss the likelihood of implanting buccal rehabilitation in the child, along with the influence of bone and dental growth of the maxillas on the stability of dental implants.

It is generally recommended to put off the buccal implanting rehabilitation until after the completion of bone and dental growth, except in cases of anodontia or severe oligodonty associated with complex syndrome.. Med Buccale Chir Buccale 2007; 13 : 219-222.

key words: child, implant, growth, oral rehabilitation, ectodermal dysplasia. médecine buccale chirurgie buccale

VOL. $13, \mathrm{~N}^{\circ} 4$ 2007

page 219 
médecine buccale chirurgie buccale

VOL. $13, \mathrm{~N}^{\circ} 4$ 2007

page 220
Pendant longtemps, les agénésies dentaires chez I'enfant étaient réhabilitées par des prothèses amovibles. Ce moyen thérapeutique répond à plusieurs attentes mais comporte des limites : défaut de stabilité en bouche et angoisse psychosociale de l'enfant et de sa famille.

Actuellement la réhabilitation buccale implantaire pourrait constituer une nouvelle option thérapeutique, bien que dans l'esprit de la majorité des praticiens la croissance osseuse inachevée constitue une limite absolue à sa mise en place chez l'enfant.

L'objet de ce travail est d'exposer les indications de l'implantologie chez l'enfant, de souligner l'intérêt particulier de la réhabilitation implantaire dans les anodonties ou les oligodonties associées à un syndrome complexe et d'établir au Maroc un espace de communication entre les différents praticiens et éducateurs impliqués notamment dans les problèmes d'agénésies dentaires multiples chez l'enfant.

\section{INTÉRÊTS ET LIMITES}

La réhabilitation prothétique à un âge précoce chez un enfant est préconisée en cas d'édentements consécutifs à un traumatisme ou une agénésie dentaire. Bien que les prothèses conventionnelles restent toujours un choix thérapeutique d'actualité, elles présentent cependant certaines limites. En effet, en plus de l'augmentation de la prévalence des lésions carieuses ainsi que la résorption de l'os alvéolaire résiduel, la réalisation d'une prothèse de substitution exige la coopération de l'enfant et de ses parents.

Pour ces mêmes indications, la prothèse sur implant constitue une nouvelle alternative à la prothèse conventionnelle avec des bénéfices esthétiques, fonctionnels et psychosociaux qui sont considérables. En effet, selon certains auteurs [2, 6, 9], l'implant permet la conservation de l'os alvéolaire, la stimulation du développement osseux ainsi que la restauration des fonctions buccales rendant à l'appareil manducateur son rôle essentiel dans la croissance craniofaciale.

Cependant, certaines réserves sont émises par d'autres ${ }^{[5,6]}$, qui soulignent que :
- Au maxillaire, le devenir d'implants placés lors de la croissance est difficilement prédictible. D'une part, une prothèse rigide s'appuyant sur des implants symétriques traversant la suture intermaxillaire peut limiter la croissance transversale. D'autre part, avec la croissance verticale, l'implant peut se retrouver enfoui dans l'os et sa portion apicale peut se trouver exposée avec le remodelage osseux du plancher sinusien.

- A la mandibule, la croissance transversale et sagittale ne semble pas être affectée par la pose d'implant. Cependant, l'inclinaison de l'implant suite à la rotation mandibulaire au cours la croissance verticale est le seul changement rapporté.

- Dans les édentations partielles, lorsque des implants sont adjacents à des dents naturelles, l'éruption de ces dernières peut entraîner un accroissement de la hauteur alvéolaire provoquant un enfouissement de l'implant qui se comporte alors comme une dent ankylosée. Le pronostic est donc plus prédictible chez les enfants édentés.

Les changements dus à la croissance peuvent entraîner l'enfouissement ou la perte de l'implant ; la mise en place de celui-ci après la puberté donnent de meilleures chances d'utilité à long terme.

\section{Intérêt particulier en cas d'anodontie ou d'oligodontie associée à des syn- dromes complexes}

Selon certains auteurs (Bergendal et coll.), le recours à la thérapeutique prothétique implantaire chez l'enfant paraît plus justifié dans les cas d'agénésies dentaires multiples, essentiellement associées à des syndromes systémiques, c'est-à-dire surtout dans les dysplasies ectodermiques.

Les dysplasies ectodermiques sont des maladies génétiques rares, qui affectent de nombreux organes issus de l'ectoderme de l'embryon. Leur incidence est estimée à 1 cas pour 100000 naissances. Elles peuvent être divisées en deux groupes cliniquement distincts :

\section{Syndrome de Christ-Siemens-Touraine ou dysplasie ectodermique anhidrotique}

C'est une pathologie liée au chromosome $X$, caractérisée par :

- Une anhidrose ou hypohidrose (pas ou peu de sudation) ; 
- Une hypoplasie dentaire (anodontie ou oligodontie) ;

- Une hypotrichose (peu de cheveux) ;

- Un front bombé, un petit nez, une ensellure nasale déprimée et une hyperpigmentation de la peau.

\section{Syndrome de Claston}

Il épargne les glandes sudoripares mais touche les dents, les cheveux et les ongles.

Ces manifestations sévères ont retenu l'attention du groupe professionnel scientifique de l'ANAES [1] (Agence Nationale d'Accréditation et d'Evaluation en Santé) qui a consacré à cette affection une réflexion profonde suivie des recommandations suivantes :

- La pose d'implant est possible sur des enfants sains atteints d'oligodontie. En l'état des connaissances actuelles, il n'y a pas de contre-indications à cette thérapeutique autres que les rares maladies affectant la croissance des os.

- La mise en place d'implant peut être envisagée à partir de l'âge de 6 ans, moment d'insertion scolaire, et jusqu'à la fin de croissance.

- La réhabilitation buccale implantaire sera envisagée après échec ou intolérance aux prothèses conventionnelles.

- La zone implantable est la région symphysaire où ne surviennent pas de modifications importantes lors de la croissance.

- Le nombre d'implant indiqué est de 2 à 4.

- La pose d'implant est exclue au maxillaire. En effet l'implant dentaire, support d'une prothèse maxillaire, se comporte comme une dent ankylosée pouvant ainsi entraîner une restriction de la croissance transversale.

En outre un implant dentaire placé dans une région postérieure subit, avec la croissance verticale par remodelage du plancher sinusien, un enfouisse- ment avec un risque d'exposition de sa portion apicale dans le sinus.

- La prise en charge pluridisciplinaire est recommandée avec une coordination entre différents spécialistes où l'odontologiste est le responsable du planning et du suivi.

- La pose d'implant chez un enfant n'est pas une solution définitive. Un implant posé chez un enfant peut être considéré comme transitoire et son retrait ne saurait être considéré comme une complication. En effet, les prothèses doivent être régulièrement ajustées, voire refaites (changement de pilier ou retrait possible de l'implant) au fur et à mesure de la croissance.

- La possibilité de recours à l'anesthésie générale ne peut être exclue.

\section{CONCLUSION}

Les enfants présentant une anodontie ou une oligodontie sévère sont rares. Ces enfants sont le plus souvent réhabilités par des prothèses amovibles conventionnelles et ne bénéficient pas de la prothèse sur implants qui constitue actuellement une option thérapeutique souvent discutée dans la littérature.

Pour cela, il semble indispensable de créer une filiale au sein de l'une de nos associations nationales de prévention regroupant les différents spécialistes impliqués dans cette affection, à savoir les implantologues, les pédiatres, les pédodontistes, les généticiens, les dermatologues, les spécialistes en imagerie maxillo-faciale et les ORL. L'objectif principal consiste à intercepter cette affection rare et à vulgariser cette thérapeutique qui apporte un confort fonctionnel et psychologique d'une autre dimension, et améliore considérablement la prise en charge de ces enfants. médecine buccale chirurgie buccale

VOL. $13, \mathrm{~N}^{\circ} 4$ 2007

page 221 


\section{RÉFÉRENCES}

1 - Agence Nationale d'Accréditation et d'Evaluation en Santé. Pose d'un implant préprothétique dans le traitement des agénésies dentaires multiples liées aux dysplasies ectodermiques ou à d'autres maladies rares (2007).

2 - Badre B, Kaoun K, Bousfiha B, Bousfiha A, Msefer S. La dysplasie ectodermique anhidrotique. A propos d'un cas clinique. J Dent Québéc 2003 ; 40 : 69-73.

3 - Bergendal B, Bergendal T, Hallonsten AL, Koch G, Kurol J, Kvint S. A mulidisciplinary approch to oral rehabilitation with osseointegrated implants in children and adolescents with multiple aplasia. Eur J Orthod 1996 ; $18: 119-29$

4 - Guckes AD, Brahim JS, McCarthy GR, Rudy S, Cooper LF. Using endosseous dental implants for patients with ectodermal dysplasia. JADA $1991 ; 122$.

5 - Guler N, Cildir S, Iseri U, Sandalli N, Dilek O. Hypohidrotic ectodermal dysplasia with bilateral impacted teeth at the coronoid process: a case rehabilated with mini dental implants. Oral Surg Oral Med Oral Pathol Oral Radiol Endod 2005 ; 99 : E34-8.

médecine

buccale

chirurgie

buccale

VOL. $13, \mathrm{~N}^{\circ} 4$ 2007
6 - Makie IC, Quaylea A. Implant in children: a case report. Endod Dent Traumatol 1993 ; 9 : 124-6.

7 - Nacht ES. Dental implant and the pediatric dental patient. Clin Pediatric Dent 1991 ; 16 : 46-47.
8 - Oesterle LJ, Cronin RJ, Ranly DM. Maxillary implants and the growing patient. Int J Oral Maxillofac Implants $1993 ; 8: 377$.

9 - Percinoto C, De Mello Vieira AE, Barbieri CM, Melhado FL, Moreira KS. Use of dental implants in children: a litterature review. Quintessence Int 2001; 32 : 381-3.

10 - Rashedi B. Prosthodontic treatment with implant fixed prosthesis for a patient with ectodermal dysplasia: a clinical report. J Prosthodont 2003 ; 12 : 198-201.

11 - Rousset-Caron M, Delfosse C, Noule M, Beauventre L, Lafforgue P. Hypodontia, oligodontia, anodontia and syndromes of developmental anomalies. Dent Med Probl $2003 ; 40: 109-15$.

12 - Rubo De Rezende ML, Monteiro Amado F. Osseointegrated implants in the oral reahbilitation of a patient with cleft lip and palate and ectodermal dysplasia: a case report. Int J Oral Maxillofac Implant 2004 ; 19 : 896-900.

13 - Smith R, Vargervik K, Kearns G, Bosh C, Koumjian J. Placement of an endosseous implant in a growing child with ectodermal dysplasia. Oral Surg Oral Med Oral Pathol $1993 ; 75$ : 669-73. 